\title{
Novel Microglomerular Structures in the Olfactory Bulb of Mice
}

\author{
Brian W. Lipscomb, ${ }^{1,2}$ Helen B. Treloar, ${ }^{2}$ and Charles A. Greer ${ }^{1,2,3}$ \\ 1/nterdepartmental Neuroscience Graduate Program, ${ }^{2}$ Department of Neurosurgery, and ${ }^{3}$ Section of Neurobiology, Yale \\ University School of Medicine, New Haven, Connecticut 06520
}

The murine olfactory system consists of two primary divisions: (1) a main olfactory system, in which olfactory sensory neurons (OSNs) located in the main olfactory epithelium (MOE) send their axons to glomeruli in the main olfactory bulb (MOB); and (2) an accessory olfactory system, in which OSNs located in the vomeronasal organ send their axons to glomeruli in the accessory olfactory bulb (AOB). In labeling studies using the lectin Ulex europaeus agglutinin (UEA), we discovered a novel subset of small neuropilar structures in the MOB that are distinct from other glomeruli both in the MOB and AOB. These "microglomeruli" are morphologically similar to MOB glomeruli in many respects: they receive innervation from processes present in the olfactory nerve layer and are isolated from other glomeruli by juxtaglomerular cells; in addition, the compartmental pattern of UEA labeling suggests the presence of UEA ${ }^{-}$processes within their neuropil. Microglomeruli contained processes that express the olfactory marker protein, a marker common to mature OSN axons. However, unlike other glomerular structures, the microglomeruli did not contain neural cell adhesion molecule-labeled processes. Within microglomeruli, UEA ${ }^{+}$processes interdigitated with $\mathrm{MAP}^{+}{ }^{+}$dendrites, some of which likely originate from interneurons, as indicated by glutamic acid decarboxylase labeling. Synaptophysin labeling in microglomeruli strongly suggested that synapses occur between UEA ${ }^{+}$ processes and dendrites. Anterograde labeling of OSNs, by injection of rhodamine-dextran into one naris, demonstrated that $\mathrm{UEA}^{+}$processes in microglomeruli originated in the MOE. The unique morphology, protein expression, and location of microglomeruli have led us to hypothesize that they represent a novel class of glomerular structures in the murine olfactory system.

Key words: glomeruli; olfactory nerve; Ulex europaeus agglutinin; NCAM
In rodents, information about the chemical composition of the environment is processed in a quartet of subsystems located in the olfactory bulb. First, information regarding the presence of volatile odorants is transduced in olfactory sensory neurons (OSNs) located in the main olfactory epithelium (MOE), which then send their axons to glomeruli located in the main olfactory bulb (MOB). In mice, the MOB contains $\sim 1800$ relatively large glomeruli ( $\sim 85 \mu \mathrm{m}$ in diameter) (Royet et al., 1998), each of which is innervated by OSNs that express 1 of 1000 olfactory receptor proteins (Ressler et al., 1993, 1994; Vassar et al., 1993, 1994; Mombaerts et al., 1996). Juxtaglomerular cells, both periglomerular interneurons as well as astrocytes, surround each glomerulus (Valverde et al., 1992).

Second, odorants that mediate species-specific social interactions such as mating, parenting, and aggression activate OSNs in the vomeronasal organ, which send their axons to glomeruli located in the accessory olfactory bulb (AOB) (Imamura et al., 1985). In mice, the AOB contains $\sim 200-300$ small glomeruli ( $\sim 30-40 \mu \mathrm{m}$ in diameter) (Belluscio et al., 1999; Rodriguez et al., 1999). In contrast to the MOB, OSNs in the AOB that express the same olfactory receptor protein target several glomeruli (for review, see Keverne, 1999; Dulac, 2000). AOB glomeruli are

\footnotetext{
Received July 30, 2001; revised Nov. 2, 2001; accepted Nov. 6, 2001.

This work was supported in part by National Institutes of Health Grants DC00210, NS10174, and DC03887. We thank Matt Wachowiak for assistance in the anterograde labeling of olfactory sensory neuron axons, Dolores Montoya for assistance with tissue preparation, and Anne Jones for administrative support.

Correspondence should be addressed to Dr. Charles A. Greer, Department of Neurosurgery, Yale University School of Medicine, 333 Cedar Street, P.O. Box 208082, New Haven, CT 06520-8082. E-mail: charles.greer@yale.edu.

Copyright (C) 2002 Society for Neuroscience $0270-6474 / 02 / 220766-09 \$ 15.00 / 0$
}

morphologically indistinct and are poorly demarcated from adjacent glomeruli (Rodriguez et al., 1999).

Third, the olfactory systems of mice and rats contain other glomerular structures, including the "atypical" glomeruli, the "necklace" glomeruli, and the glomeruli of the modified glomerular complex (MGC). The relationship of these glomerular structures to each other is unclear (Greer et al., 1982; Shinoda et al., 1993; Ring et al., 1997; Baker et al., 1999; Weruaga et al., 2001), but on the whole they display a morphological and immunocytochemical profile that is distinct from that of $\mathrm{MOB}$ and $\mathrm{AOB}$ glomeruli (Shinoda et al., 1989, 1993). Necklace glomeruli and glomeruli in the MGC are similar in size to MOB glomeruli (Weruaga et al., 2001) and are innervated by OSNs that originate in the MOE (Juilfs et al., 1997). Although the physiological significance of these structures is unclear, the MGC has been implicated in the suckling response of rats (Greer et al., 1982; Risser and Slotnick, 1987).

Fourth, morphologically distinct OSNs in the septal organ of mice and rats have been shown to innervate some glomeruli in the MOB (Pedersen and Benson, 1986; Giannetti et al., 1992) (M. $\mathrm{Ma}$, personal communication). The functional significance of the glomeruli receiving input from the septal organ and their relationship to other MOB glomeruli remains unclear.

In the present study we identify a set of small $(\sim 30 \mu \mathrm{m}$ in diameter) neuropilar structures, the microglomeruli, which are located throughout the MOB but appear to be unrelated to main, accessory, necklace, atypical, or MGC glomeruli or to glomeruli innervated by the septal organ. For example, microglomeruli do not contain axons that express neural cell adhesion molecule (NCAM), a marker common to axons found in most glomeruli 
(Yamashita et al., 1998). However, microglomeruli do share other characteristics with MOB glomeruli: they contain axons that express olfactory marker protein (OMP), are clearly demarcated by juxtaglomerular cells, have distinct zones of dendritic compartmentalization, and appear to contain synapses. Axons that innervate microglomeruli originate in the MOE, as demonstrated by anterograde labeling of microglomeruli after nasal lavage with a labeled dextran. This final feature suggests that microglomeruli are quite different from the nidi, small neuropilar structures described in the MOB of the musk shrew, which lack input from the MOE (Kosaka and Kosaka, 1999, 2001). The unique morphology and location of microglomeruli have led us to the hypothesis that they represent a novel class of glomerular organization in the murine olfactory system.

\section{MATERIALS AND METHODS}

\section{Immunocytochemistry}

Tissue preparation. CD1 female mice $(n=20)$ that were $30-60 \mathrm{~d}$ old were used. The care and procedures used were reviewed and approved by the Yale Animal Care and Use Committee. The mice were anesthetized with sodium pentobarbital $(100 \mathrm{mg} / \mathrm{kg}$ Nembutal; Abbott Laboratories, North Chicago, IL) and then fixed by transcardiac perfusion with PBS $(0.1 \mathrm{M}$ phosphate buffer and $0.9 \% \mathrm{NaCl}, \mathrm{pH} 7.4$ ) containing $0.1 \%$ heparin sulfate. After clearing of the liver, the animal was perfused for $5 \mathrm{~min}$ with $4 \%$ paraformaldehyde (PFA) in PBS, pH 6.5, and then for $15 \mathrm{~min}$ in $4 \%$ PFA in PBS, pH 10.5. It should be noted that this perfusion protocol was crucial for visualization of microglomeruli. Perfusion solely with $4 \%$ PFA, pH 7.4, revealed Ulex europaeus agglutinin (UEA)-labeled axons in MOB glomeruli, but this perfusion was insufficient to preserve the carbohydrate epitope labeled by UEA in microglomeruli. Brains were removed and immersed in perfusate for $24 \mathrm{hr}$ at $4^{\circ} \mathrm{C}$. All tissue was mounted in $2 \%$ agarose and sectioned on a Pelco (Redding, CA) 101 Vibratome into $50 \mu \mathrm{m}$ sections.

Single-labeled fluorescent tissue. For single-labeled fluorescent studies, tissue was blocked for 30 min with $2 \%$ bovine serum albumin (BSA) and $0.3 \%$ Triton $\mathrm{X}-100$ in TBS $(0.1 \mathrm{M}$ Tris buffer and $0.9 \% \mathrm{NaCl}, \mathrm{pH} 7.4)$. The tissue was then incubated in $10 \mu \mathrm{g} / \mathrm{ml}$ biotin-labeled UEA (EY Laboratories, San Mateo, CA) for 2 hr. After several TBS washes, the tissue was incubated in Alexa-568-conjugated streptavidin (1:200; Molecular Probes, Eugene, OR) for $1 \mathrm{hr}$. The tissue was washed with TBS and mounted in Vectashield (Vector Laboratories, Burlingame, CA). For single-labeling studies in which surrounding nuclei were visualized (compare Fig. 2), the tissue was processed as described above, but UEA labeling was visualized with Alexa-488-conjugated streptavidin (1:200; Molecular Probes) and mounted in Vectashield containing propidium iodide (Vector Laboratories).

Although $10 \mu \mathrm{g} / \mathrm{ml}$ UEA was used in all experiments for consistency, UEA labeling of microglomeruli was observed at concentrations as low as $1 \mu \mathrm{g} / \mathrm{ml}$. Lectin labeling was blocked with the addition of exogenous sugars. UEA labeling was blocked with $0.1 \mathrm{M}$ L-fucose but not with either $0.1 \mathrm{M}$ D-fucose or $0.4 \mathrm{M} \mathrm{N}$-acetylgalactosamine (data not shown).

Double-labeled fluorescent tissue. For double-labeling studies, the tissue was processed as for single-labeled fluorescence, but cocktails of primary and secondary antibodies were used. Tissue was incubated in a cocktail of biotin-conjugated UEA $(10 \mu \mathrm{g} / \mathrm{ml}$; EY Laboratories) together with (1) a rabbit polyclonal antibody to NCAM (1:200; Chemicon, Temecula, CA); (2) a goat polyclonal antibody to OMP (1:250; courtesy of Frank Margolis, Baltimore, MD); (3) a mouse monoclonal antibody to MAP2 (1:100; Sigma, St. Louis, MO); (4) a rabbit polyclonal antibody to glutamic acid decarboxylase (GAD)-67 (1:1000; Chemicon); or (5) a rabbit polyclonal antibody to human synaptophysin (1:100; Dako, Glostrup, Denmark). After washing, the tissue was incubated in Alexa568-conjugated streptavidin (1:200; Molecular Probes) together with (1) an Alexa-488-conjugated goat anti-rabbit IgG [heavy and light chain (H + L)] antibody (1:200; Molecular Probes); (2) an Alexa-488-conjugated donkey anti-goat antibody (1:100; Molecular Probes); (3) an Alexa-488conjugated goat anti-mouse $\operatorname{IgG}(\mathrm{H}+\mathrm{L})$ antibody (1:100; Molecular Probes); (4) an Alexa-488-conjugated goat anti-rabbit $\operatorname{IgG}(\mathrm{H}+\mathrm{L})$ antibody (1:200; Molecular Probes); or (5) an Alexa-488-conjugated goat anti-rabbit IgG $(\mathrm{H}+\mathrm{L})$ antibody (1:100; Molecular Probes), respectively. After washing, the tissue was mounted in Vectashield as described above.

\section{Lavage of naris with dextran}

CD1 female mice $(n=8)$ that were $30-40$ d old were anesthetized with $100 \mathrm{mg} / \mathrm{kg}$ ketamine. Once the mice were under anesthesia, $2 \mu \mathrm{l}$ of $0.25 \%$ Triton X-100 dissolved in sterile water was injected into one naris. After $3 \mathrm{~min}, 8 \mu \mathrm{l}$ of $8 \%$ Texas Red-labeled dextran (3000 molecular weight; Molecular Probes) in a $0.25 \%$ Triton X-100 solution in sterile water was slowly injected into the naris for $10 \mathrm{~min}$. The animal was allowed to recover for $20 \mathrm{~min}$ and the procedure was then repeated on the other naris. The animal was allowed to recover after the procedure and was killed 5-7 d later and perfused; the tissue was prepared for immunocytochemistry as detailed above.

The tissue was blocked with $2 \%$ BSA and $0.3 \%$ Triton X-100 in TBS for $30 \mathrm{~min}$. The sections were then incubated in $10 \mu \mathrm{g} / \mathrm{ml}$ biotinconjugated UEA (EY Laboratories) and a mouse monoclonal IgG1 antibody to NCAM (1:200; Sigma) in the same blocking solution as above for $2 \mathrm{hr}$ at room temperature. After three $10 \mathrm{~min}$ washes with $0.3 \%$ Triton X-100 in TBS, the tissue was incubated in Alexa-488-conjugated streptavidin (1:100; Molecular Probes) and a Cy5-conjugated goat antimouse antibody (1:100; Jackson ImmunoResearch, West Grove, PA) in the above blocking solution for $1 \mathrm{hr}$. Tissue was washed twice in $0.3 \%$ Triton X-100 in TBS and once in TBS and then mounted in $40 \%$ glycerol.

\section{Image acquisition and processing}

Images were acquired using a Bio-Rad (Hercules, CA) 600 scanning confocal microscope. All images were processed using Corel Draw 8.0 and Corel Photo Paint 8.0 (Corel Inc., Ottawa, Ontario, Canada). All images were adjusted for contrast and brightness to equilibrated light levels. Images were cropped, resized, and rotated for purposes of presentation. Images of double- and triple-labeled tissue were adjusted to equalize staining intensity levels and background in the images. In no case was the content of images altered.

\section{RESULTS}

Labeling the mouse olfactory bulb with UEA revealed a novel subset of neuropilar structures. Although these structures were found in the glomerular layer of the MOB, they were similar in size to glomeruli observed in the AOB. Because of their dissimilarity to the glomeruli described previously, we have coined the term microglomerulus and the plural microglomeruli to refer to this anatomical feature. To characterize microglomeruli, we have examined the expression of proteins typically associated with MOB glomeruli and investigated the relationship between UEAlabeled $\left(\mathrm{UEA}^{+}\right)$axons and potential synaptic targets within the microglomerular neuropil.

\section{Microglomeruli in the main olfactory bulb}

The binding patterns of various lectins have been examined extensively in the olfactory system. In many species, lectins that preferentially bind different carbohydrate epitopes label subsets of OSNs in the MOE and OSN axons in glomeruli (for review, see Plendl and Sinowatz, 1998). We demonstrate that the lectin UEA labels a subset of glomeruli in the MOB (Fig. 1A) and all glomeruli in the AOB (data not shown). UEA also intensely labeled very small neuropilar structures, usually located in the deep glomerular layer, which did not appear to be associated with MOB glomeruli (Fig. 1B). These neuropilar structures, or microglomeruli, averaged $\sim 30 \mu \mathrm{m}$ in diameter. However, microglomeruli varied greatly, ranging in size from $\sim 20-50 \mu \mathrm{m}$ and in morphology from spheres to narrow cylinders (compare especially Fig. $2 A-F)$. UEA ${ }^{+}$processes within microglomeruli exhibited a compartmentalized pattern reminiscent of that observed in MOB glomeruli.

Microglomeruli were often innervated by processes that originated in the olfactory nerve layer (ONL). These processes clearly passed through the main glomerular layer before densely arborizing within microglomeruli. In some cases processes that targeted 

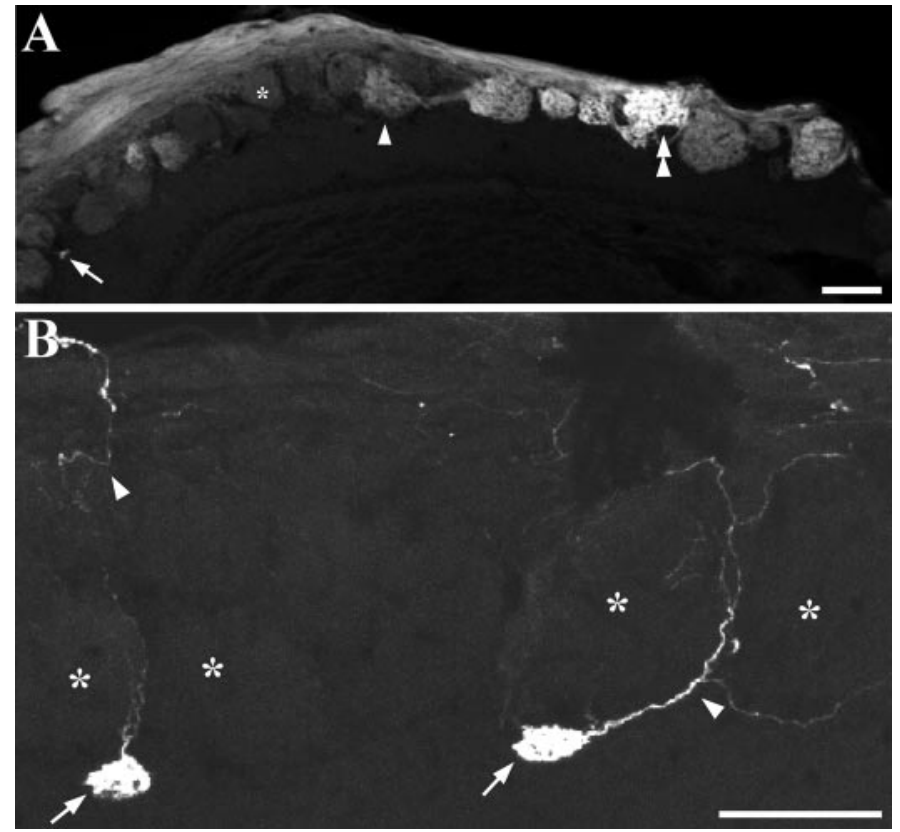

Figure 1. UEA labeling in the olfactory bulb of a mouse. $A$, UEA labeled some glomeruli intensely (double arrowhead), whereas other glomeruli were labeled less strongly (single arrowhead). Unlabeled glomeruli were also observed (asterisk). Small neuropilar structures intensely labeled by UEA were observed in the glomerular layer (arrow, see below). $B$, UEA intensely labeled a subset of small neuropilar structures (arrows) that we termed microglomeruli. In this compressed $\mathrm{Z}$ series, what appear to be axons (arrowheads) can be seen weaving between glomeruli (asterisks) and then entering microglomeruli. Scale bars: $A, 100 \mu \mathrm{m} ; B, 50 \mu \mathrm{m}$.

microglomeruli followed several paths toward their goal, fasciculating into larger bundles as they approached the microglomerulus (compare Figs. $1 B, 3 C$ ). In their path to microglomeruli, these processes often curled closely around MOB glomeruli that contained no UEA labeling (Fig. $1 B$ ).

$\mathrm{UEA}^{+}$microglomeruli were common in the adult olfactory bulb, with several observed in a single $50-\mu$ m-thick coronal section (Fig. 2). Based on counts from several animals, we estimate that each adult olfactory bulb contains $\sim 100-150$ UEA $^{+}$microglomeruli (data not shown). Reconstruction of olfactory bulbs through serial sections suggested that microglomeruli were not the result of glancing sections through the periphery of larger glomeruli. Rather, microglomeruli were self-contained, and their neuropil was not continuous with adjacent glomeruli. Using propidium iodide to visualize nuclei, it became evident that the microglomerular neuropil was isolated from the neuropil of adjacent glomeruli. As in traditional MOB glomeruli, a ring of juxtaglomerular cells separates microglomeruli from other glomerular structures and from the external plexiform layer (EPL) (Fig. 2A-F) (Kosaka et al., 1998). The interior of the microglomerular neuropil was devoid of nuclei.

\section{Microglomeruli and classic OSN markers}

Although microglomeruli resembled MOB glomeruli morphologically in several respects, we wished to determine whether UEA ${ }^{+}$ processes in microglomeruli contained proteins typically found in OSNs. In developing (Terkelsen et al., 1989; Gong and Shipley, 1996; Treloar et al., 1997) and mature (Yamashita et al., 1998) mice, OSNs that target the MOB and AOB strongly express NCAM in their cell bodies and axons. To assess the presence of NCAM in microglomeruli, we double-labeled olfactory bulbs
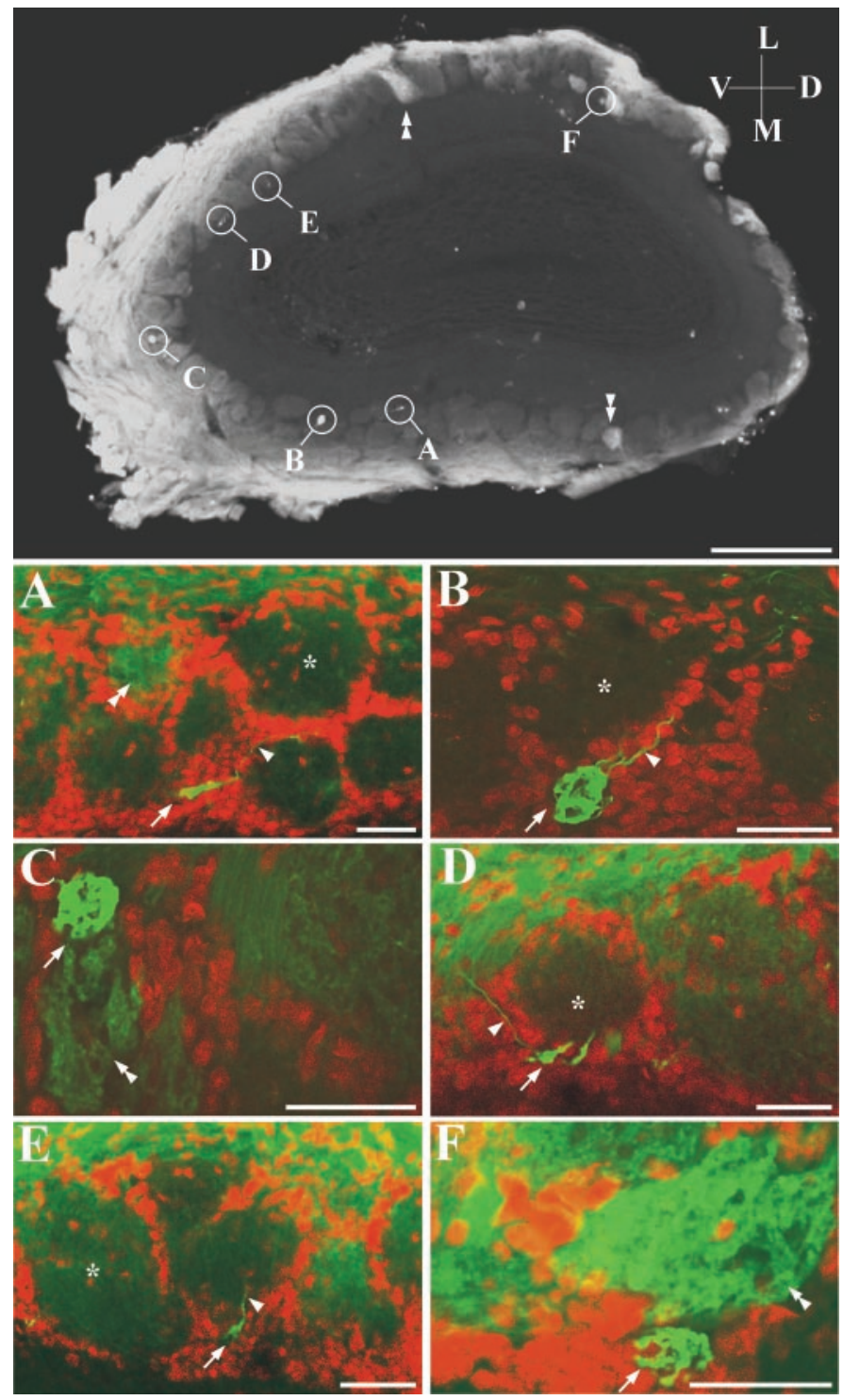

Figure 2. Microglomeruli in a 50- $\mu \mathrm{m}$-thick coronal section of the olfactory bulb. Intense UEA labeling was observed in several microglomeruli (circles $A-F$ ) as well as in some traditional glomeruli (double arrowheads). $A-F$, The microglomeruli circled above displayed intense UEA labeling (green, arrows) and were surrounded by propidium iodide-labeled nuclei (red). $\mathrm{UEA}^{+}$processes were often observed traveling around labeled nuclei and other glomeruli to target microglomeruli (arrowheads in $A, B$, $D$, and $E$ ). Adjacent traditional glomeruli often contained no UEA labeling (e.g., asterisks), although some microglomeruli were found near $\mathrm{UEA}^{+}$glomeruli (double arrowheads). Scale bars: top panel, $500 \mu \mathrm{m} ; A-F$, $50 \mu \mathrm{m}$.

with UEA and NCAM (Fig. 3). As expected, NCAM labeling was prominent in the ONL and in MOB glomeruli but absent from other layers of the olfactory bulb. Surprisingly, $\mathrm{UEA}^{+}$microglomeruli were not labeled by NCAM. This was true both in the main body of microglomeruli (Fig. $3 A-E$, arrows) and in the processes that targeted microglomeruli (Fig. $3 A-C$, arrowheads). However, NCAM and UEA double labeling was observed in MOB glomeruli (Fig. 3B, double arrowhead), suggesting that the lack of NCAM labeling in microglomeruli was not attributable to interference by the UEA lectin. $\mathrm{UEA}^{+} / \mathrm{NCAM}^{-}$microglomeruli were typically located below the glomerular layer but occasionally could be found within the glomerular layer wedged between 

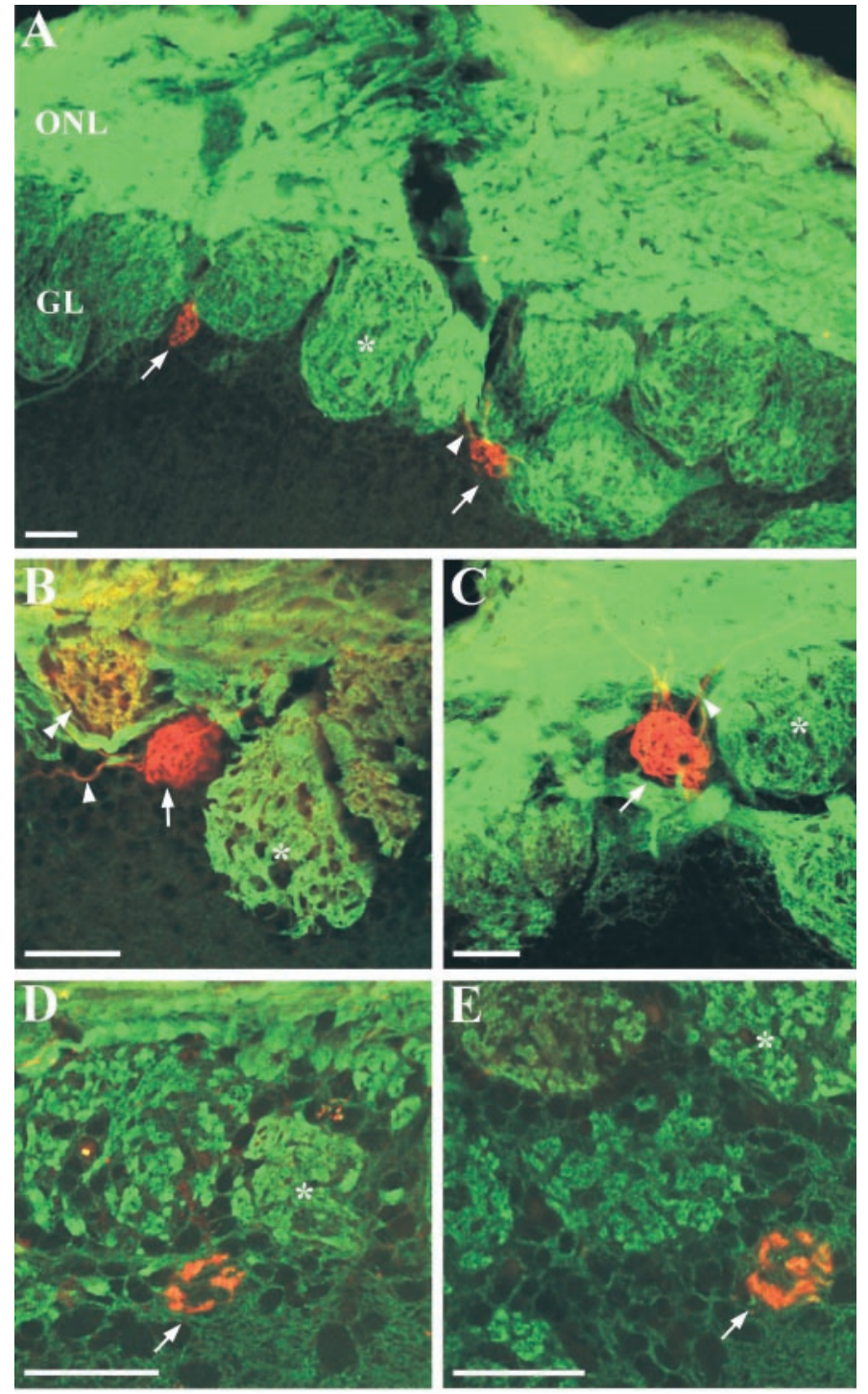

Figure 3. $\mathrm{UEA}^{+}$microglomeruli are not labeled by NCAM. $A$, NCAM labeling (green) was prominent in OSN axons in the ONL and glomeruli in the glomerular layer (GL; e.g., asterisk). $\mathrm{UEA}^{+}$microglomeruli (red, arrows) were not labeled with NCAM. A UEA ${ }^{+}$axon is seen approaching a microglomeruli (arrowhead). $B-E$, Examples of UEA microglomeruli not labeled by NCAM. OSN axons that target traditional glomeruli were $\mathrm{NCAM}^{+}$( green, asterisk) and were also sometimes labeled with UEA ( $B$, yellow, double arrowhead). $\mathrm{UEA}^{+}$microglomeruli were $\mathrm{NCAM}^{-}$(arrows). $\mathrm{UEA}^{+}$axons were seen approaching microglomeruli (arrowheads). Scale bars, $50 \mu \mathrm{m}$.

$\mathrm{NCAM}^{+}$MOB glomeruli (Fig. 3B,C). Although microglomeruli were not labeled by NCAM, they were labeled by OMP, a classic marker of mature OSNs in both the MOB and AOB (Margolis, 1982; Baker et al., 1989). OMP labeling was present on $\mathrm{UEA}^{+}$ processes within microglomeruli as well as on $\mathrm{UEA}^{+}$processes in the ONL, which terminated in microglomeruli (Fig. 4A). Microglomeruli displayed a compartmentalized OMP staining pattern similar to that observed in traditional MOB glomeruli (Fig. 4A-C) (Kasowski et al., 1999).

\section{Characterization of the microglomerular neuropil}

In traditional MOB glomeruli, OSN axons form dense compartments within the glomerulus. These compartments interdigitate with similarly sized compartments made up primarily of dendrites
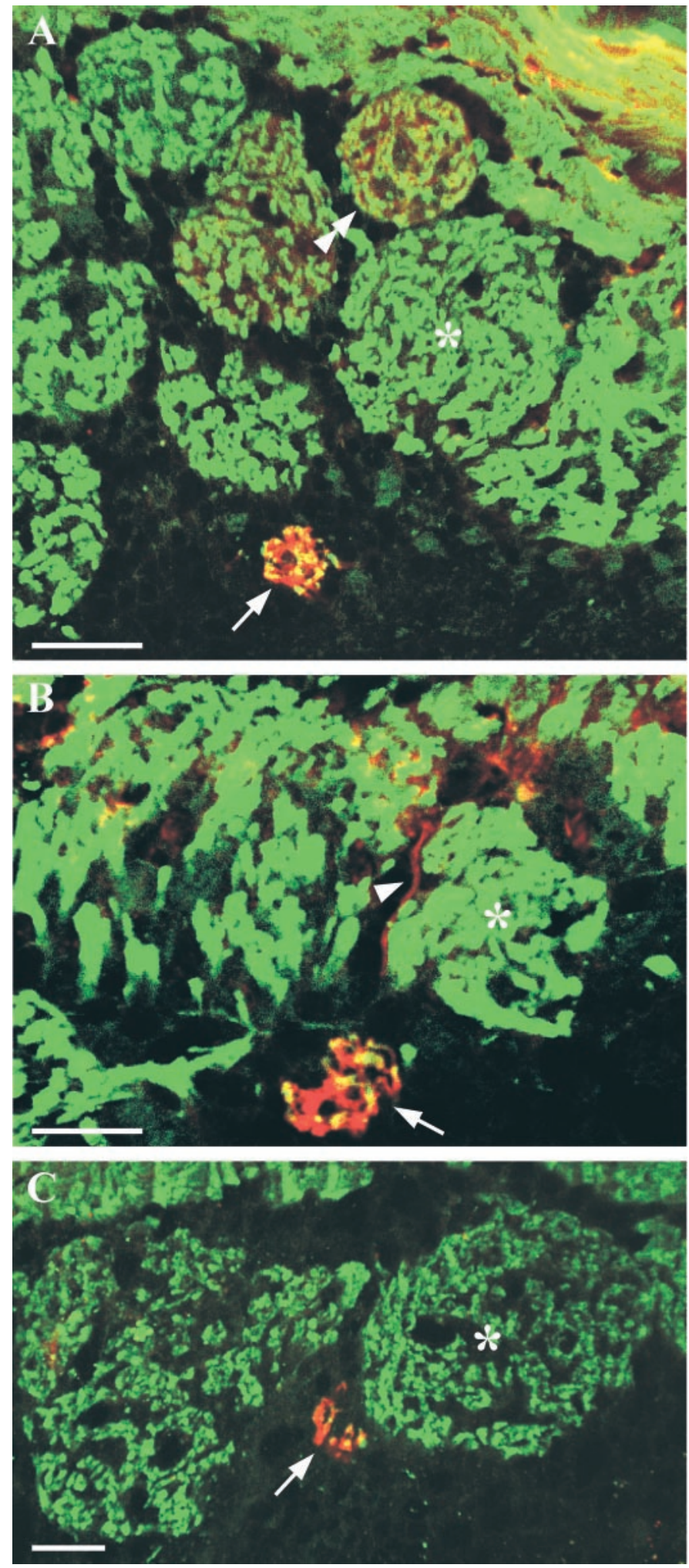

Figure 4. $\mathrm{UEA}^{+}$microglomeruli contain OMP labeling. $A-C$, OMP labeling (green) was prominent in main olfactory glomeruli (asterisk), some of which contained UEA labeling ( yellow, double arrowhead). UEA labeling in microglomeruli (red, arrows) colocalized with OMP labeling (demonstrated by yellow labeling in their neuropil). A UEA ${ }^{+}$axon was seen approaching a microglomerulus (arrowhead). Scale bars: $A, 50 \mu \mathrm{m}$; $B, C, 25 \mu \mathrm{m}$. 

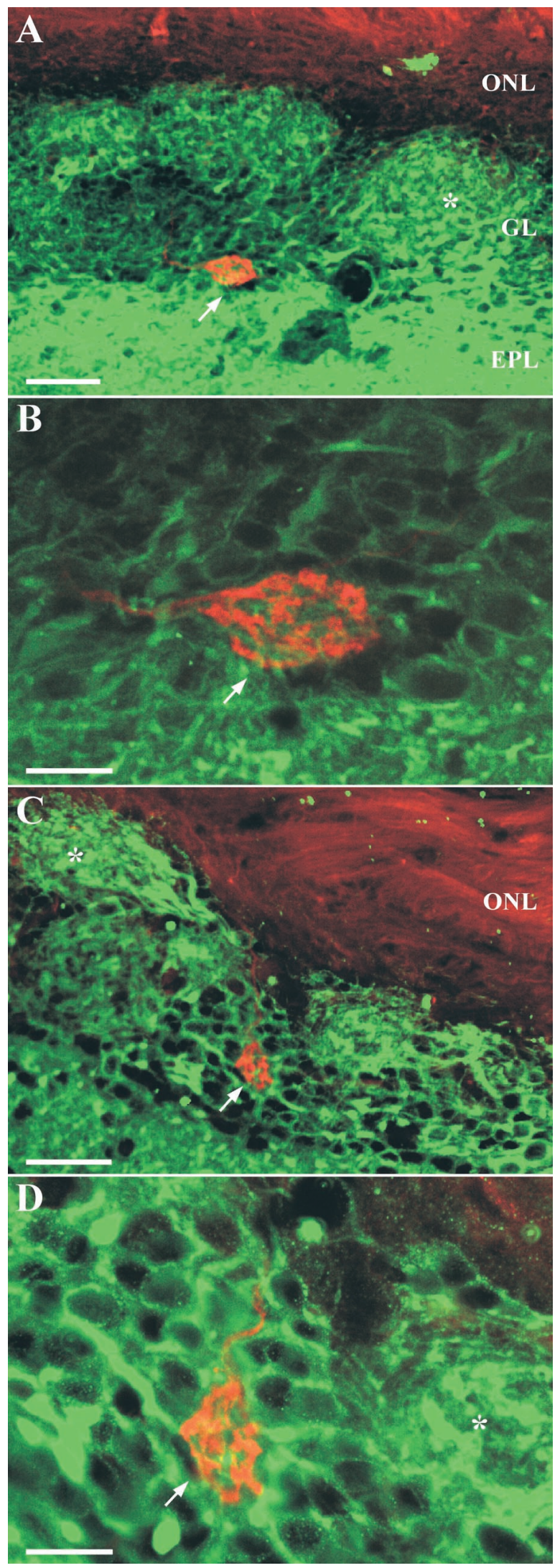

Figure 5. The microglomerular neuropil contains dendrites. $A-D$, $\mathrm{UEA}^{+}$axons (red) in microglomeruli (arrow) interdigitate with MAP2 ${ }^{+}$ dendrites (green). MAP2 labeling was also prominent in traditional glomeruli (e.g., asterisks). A, MAP2 staining was absent from the ONL but was prominent in the glomerular layer $(G L)$ and the EPL. A micro- originating from projection neurons and interneurons (Kasowski et al., 1999). Because microglomeruli exhibit a compartmentalized innervation pattern similar to that seen in MOB glomeruli (compare Fig. 3D,E), we have postulated that axons in microglomeruli also interdigitate with dendrites. To address this issue, we double-labeled olfactory tissue with UEA and MAP2, a cytoskeletal protein restricted to dendrites. $\mathrm{MAP}^{+}$dendrites were present in the glomerular layer and dendrite-rich external plexiform layer; however, as expected, they were absent from the ONL (Fig. 5A-D) (Kasowski et al., 1999; Kim and Greer, 2000). In microglomeruli, $\mathrm{UEA}^{+}$processes in axonal compartments interdigitated with MAP2 ${ }^{+}$dendrites in dendritic compartments (Fig. $5 A-D)$.

OSN axons in MOB glomeruli form synapses with mitral/tufted cells, the projection neurons of the olfactory bulb, and interneurons, which are primarily GABAergic (Ribak et al., 1977; Gabellec et al., 1980). We used an antibody to GAD, an enzyme crucial to GABA synthesis (Lernmark, 1996), to label interneurons in the olfactory bulb. Double labeling olfactory tissue with UEA and $\mathrm{GAD}$ revealed that $\mathrm{UEA}^{+}$processes interdigitated with $\mathrm{GAD}^{+}$processes in microglomeruli. $\mathrm{GAD}^{+}$processes were also seen in $\mathrm{UEA}^{+}$MOB glomeruli, as well as in MOB glomeruli that contained no UEA labeling (compare Fig. 6A,B). Indeed, the density of $\mathrm{GAD}^{+}$processes in microglomeruli was similar to that observed in MOB glomeruli. Significantly, no $\mathrm{UEA}^{+} / \mathrm{GAD}^{+}$ processes were observed, suggesting that no $\mathrm{UEA}^{+}$processes originated from interneurons. The presence of $\mathrm{MAP}^{+}$and $\mathrm{GAD}^{+}$processes in microglomeruli suggests that in microglomeruli $\mathrm{UEA}^{+}$axons interact with dendrites. It is unclear whether all $\mathrm{MAP}^{+}$processes within the microglomerular neuropil originate from interneurons or whether dendrites that originate from projection neurons are also present. However, because areas of the microglomeruli appeared not to be labeled by either UEA or GAD, it seems reasonable to expect the presence of a population of non-GABAergic dendrites within microglomeruli (compare Fig. $6 A, B)$.

The proximity of $\mathrm{UEA}^{+}$processes and dendrites in microglomeruli suggested the existence of synapses within the microglomerular neuropil. To further examine this possibility, olfactory tissue was double-labeled with UEA and synaptophysin.

As expected, synaptophysin intensely labeled the glomerular layer and EPL but was absent from the ONL (Kasowski et al., 1999). In microglomeruli, synaptophysin labeling was closely associated with $\mathrm{UEA}^{+}$processes (Fig. $6 C, D$, yellow). The colocalization of UEA and synaptophysin suggests that $\mathrm{UEA}^{+}$processes make synapses within microglomeruli. Because synaptophysin labeling was also prominent in the interior of the dendritic areas of the microglomerular neuropil (Fig. 6C,D, green), it seems reasonable to suggest that dendrodendritic synapses occur within microglomeruli.

\section{$\mathrm{UEA}^{+}$axons within microglomeruli originate in the MOE}

Although $\mathrm{UEA}^{+}$processes that innervate microglomeruli could be traced to the ONL before entering microglomeruli and were $\mathrm{OMP}^{+}$, the lack of NCAM labeling in microglomeruli and their

$\leftarrow$

glomerulus is seen at the interface of the GL and the EPL (arrow). $B$, $\mathrm{UEA}^{+}$processes in the microglomerulus seen in $A$ (arrow) interdigitate with $\mathrm{MAP} 2^{+}$dendrites. $C$, A microglomerulus in the glomerular layer (arrow). $D, \mathrm{UEA}^{+}$processes in the microglomerulus seen in $C$ (arrow) interdigitate with $\mathrm{MAP} 2^{+}$dendrites. Scale bars: $A, C, 50 \mu \mathrm{m} ; B, D, 10 \mu \mathrm{m}$. 

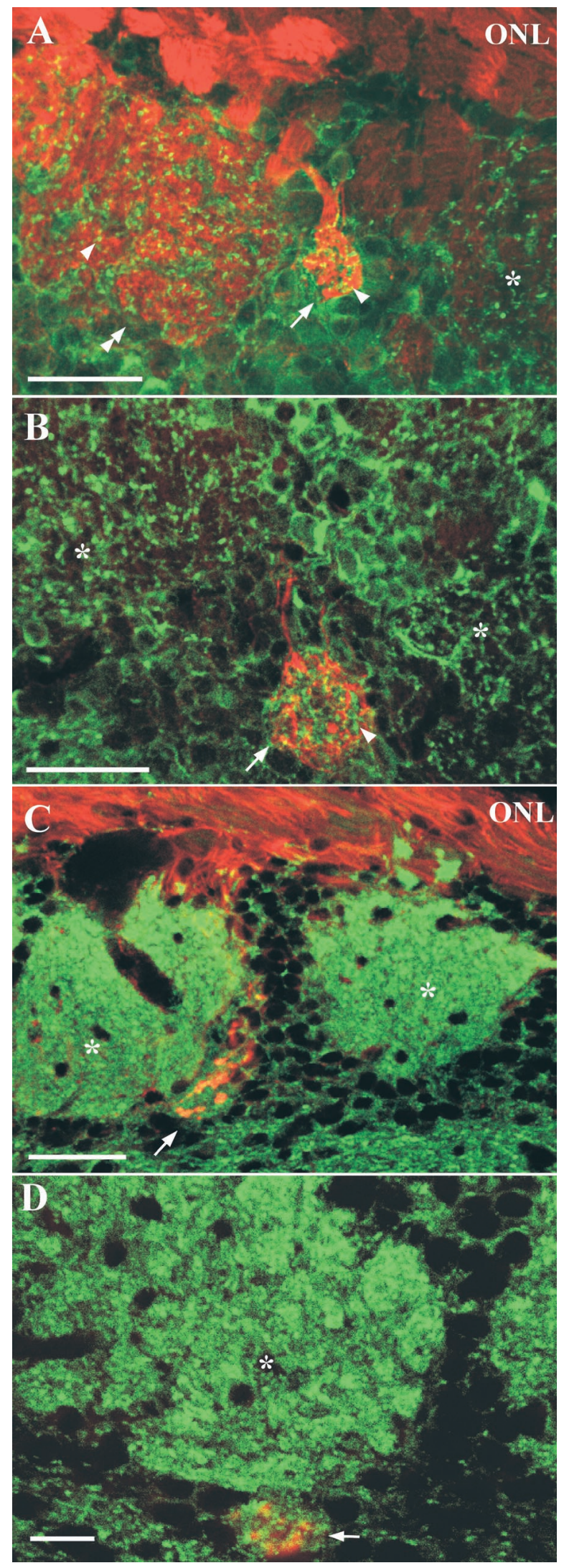

Figure 6. The microglomerular neuropil contains interneurons and synapses. $A, B, \mathrm{UEA}^{+}$microglomeruli and GAD-labeled interneuron dendrites. $\mathrm{UEA}^{+}$microglomeruli (arrows), $\mathrm{UEA}^{+} \mathrm{MOB}$ glomeruli (double arrowhead), and MOB glomeruli containing no UEA labeling (asterisks) displayed numerous $\mathrm{GAD}^{+}$puncta (arrowheads). $C, D$, $\mathrm{UEA}^{+}$microglo- unusual morphology raised the possibility that axons that target microglomeruli may not originate in the MOE. To determine whether microglomeruli contained axons that originate in the MOE, we chose to label OSNs anterogradely in the MOE. Briefly, rhodamine-labeled dextran beads in a solution containing a detergent were injected into the naris of an adult mouse; after 5-7 $\mathrm{d}$, the tissue was double-labeled with UEA and NCAM to assess the appearance of rhodamine-labeled dextran in MOB glomeruli and microglomeruli. In general, rhodamine labeling in the olfactory bulb was sporadic. Glomeruli on the dorsal surface of the olfactory bulb were strongly labeled, but throughout other areas of the olfactory bulb labeling was often absent or was restricted to a few processes. The AOB contained very little rhodamine labeling, suggesting a limited penetration of the vomeronasal organ during the naris lavage. No labeling by rhodamine-labeled dextran was observed outside the ONL or glomeruli. Thus, in general, labeling of OSNs that project to the olfactory bulb with rhodamine-labeled dextran appeared to be a conservative measure of the afferent innervation of the olfactory bulb.

Nevertheless, several $\mathrm{NCAM}^{-}$microglomeruli contained rhodamine-labeled dextran, as did adjacent MOB glomeruli (Fig. $7 A, B)$. Dextran labeling in these microglomeruli was light and punctate, and dextran labeling in the adjacent MOB glomeruli was of a similar quality. Because the AOB showed no evidence of consistent or significant labeling, the most parsimonious interpretation of these data is that microglomeruli received afferent innervation from OSNs located in the MOE.

The presence of rhodamine-labeled dextran in the neuropil of $\mathrm{NCAM}^{-}$microglomeruli indicated that the axons that innervate microglomeruli originate in the MOE. However, it was unclear whether the lack of NCAM labeling observed in axons within microglomeruli extended to cell bodies located in the MOE. To confirm the existence of $\mathrm{NCAM}^{-}$OSNs in the MOE, we doublelabeled MOE tissue with UEA and NCAM. Postnatal day 6 (P6) mice were used in this investigation to more easily obtain tissue sections from the MOE. At P6, numerous $\mathrm{UEA}^{+} / \mathrm{NCAM}^{-} \mathrm{mi}^{-}$ croglomeruli are present in the $\mathrm{MOB}$ and are morphologically similar to those seen in mature mice (Fig. 7C). In MOE tissue from the same animal, UEA ${ }^{+}$OSNs were spread throughout the MOE. In a subset of OSNs intensely labeled by UEA, there was an absence of NCAM labeling on their cell bodies, dendrites, and cilia (Fig. 7D). $\mathrm{UEA}^{+} / \mathrm{NCAM}^{-}$OSNs were surrounded by OSNs that displayed NCAM labeling (Fig. 7D, arrow). There was no zonal organization in the distribution of $\mathrm{UEA}^{+} / \mathrm{NCAM}^{-} \mathrm{OSNs}$. Although $\mathrm{UEA}^{+} / \mathrm{NCAM}^{-}$OSNs were found in all of the turbinates and along the length of the septal epithelium, they were rare, and it was typical to find only one example in a $20 \mu \mathrm{m}$ section.

\section{DISCUSSION}

Using the lectin UEA, we have identified a previously unrecognized neuropilar structure, the microglomerulus, in the MOB of adult mice. Morphologically, microglomeruli were distinctly smaller than MOB glomeruli and were typically located at the border of the glomerular layer and the EPL. UEA ${ }^{+}$axonal

$\leftarrow$

meruli and synaptophysin labeling. Synaptophysin labeling (green) was absent from the ONL but prominent in MOB glomeruli (asterisks). In microglomeruli (arrows), $\mathrm{UEA}^{+}$axons were double-labeled with synaptophysin (yellow). Dendritic areas within microglomeruli were also labeled by synaptophysin (green). Scale bars: $A-C, 50 \mu \mathrm{m} ; D, 10 \mu \mathrm{m}$. 

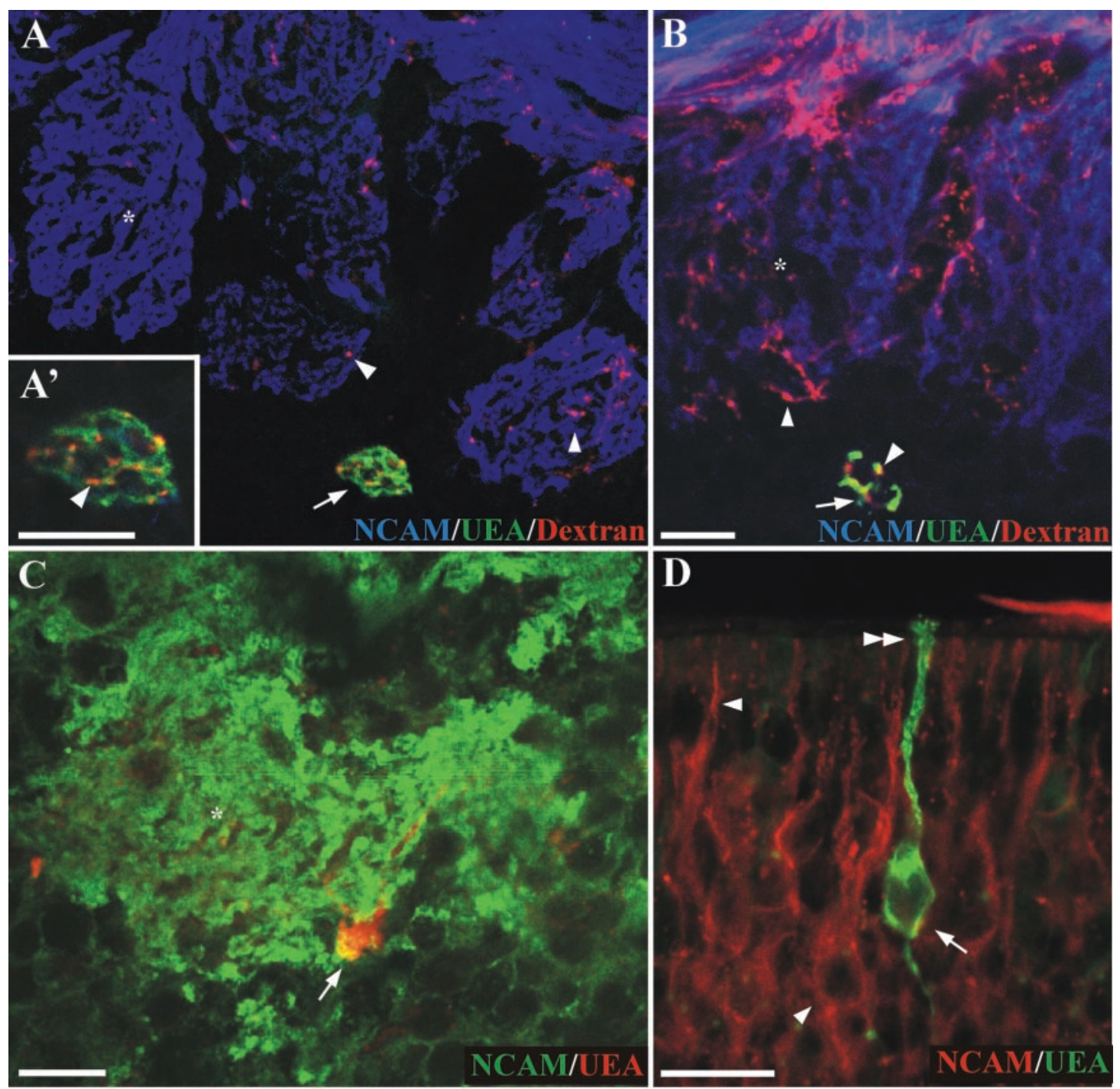

Figure 7. $\mathrm{UEA}^{+}$axons within microglomeruli originate in the MOE. $A$, Rhodamine-labeled dextran (red) injected into the naris was anterogradely transported into MOB glomeruli (arrowheads) and microglomeruli (arrow). Some glomeruli were not labeled by dextran (asterisk). $A^{\prime}$, Higher magnification of microglomerulus in $A$. Rhodomine-labeled puncta (red, arrowhead) can be seen in $\mathrm{UEA}^{+} / \mathrm{NCAM}^{-}$(green) microglomerulus. $B$, The neuropil of $\mathrm{NCAM}^{+}$glomeruli (blue, asterisk) and $\mathrm{NCAM}^{-}$microglomeruli (green, arrow) contained small rhodamine-labeled puncta (red, arrowheads). $C$, $\mathrm{UEA}^{+}$microglomeruli (arrow) and a UEA ${ }^{-}$MOB glomerulus (asterisk) were observed in mice at P6. $D$, In the MOE of P6 mice, some UEA ${ }^{+} \mathrm{OSNs}^{-}$ were $\mathrm{NCAM}^{-}$(arrow). UEA labeling in these neurons was prominent in the cilia (double arrowhead). Numerous NCAM ${ }^{+} \mathrm{OSNs}$ were also observed (e.g., arrowheads). Scale bars: $A, 50 \mu \mathrm{m} ; A^{\prime}, B-D, 25 \mu \mathrm{m}$.

processes that innervate microglomeruli did not express NCAM, a cell adhesion molecule common to nearly all OSN axons. However, in other respects microglomeruli appeared to be mature, functional olfactory structures: (1) $\mathrm{UEA}^{+}$processes within microglomeruli were also $\mathrm{OMP}^{+}$; (2) within microglomeruli, MAP2 labeling interdigitated with $\mathrm{UEA}^{+}$processes, demonstrating the apposition of axons and dendrites; (3) numerous GAD ${ }^{+}$ processes within microglomeruli indicated the presence of dendrites originating from interneurons; (4) synaptophysin labeling associated with $\mathrm{UEA}^{+}$processes indicated the presence of functional synapses within microglomeruli; and (5) afferent processes that innervate microglomeruli originate in the MOE.

\section{Are microglomeruli novel structures?}

Given the noted variety of olfactory structures, we considered the possibility that microglomeruli represented a structure homologous to one seen in other mammals or were unrecognized mem- bers of a family of murine olfactory structures that has been described previously. Microglomeruli were similar in size and location to nidi, olfactory structures found in the laboratory musk shrew (Kosaka and Kosaka, 1999, 2001). Like microglomeruli, nidi were found at the interface of the glomerular layer and the EPL, adjacent to traditional MOB glomeruli. However, whereas nidi appeared to be as numerous as MOB glomeruli, UEA ${ }^{+}$ microglomeruli were much less numerous than MOB glomeruli. Significantly, nidi appeared to receive no innervation from the $\mathrm{MOE}$ and consisted primarily of $\mathrm{GAD}^{+}$processes. In contrast, $\mathrm{UEA}^{+}$processes in microglomeruli originated in the MOE, and $\mathrm{GAD}^{+}$processes made up a small percentage of the microglomerular neuropil. Although rats and mice contain several nontraditional glomerular structures, including the MGC, atypical and necklace glomeruli, and septal organ glomeruli, these structures are all similar in size to MOB glomeruli and are confined to small regions of the bulb. Microglomeruli, in contrast, were found 
throughout the olfactory bulb and were much smaller than previously identified nontraditional murine glomeruli.

We have considered the possibility that microglomeruli are either nascent developing glomeruli or glomeruli that are atrophying and undergoing degeneration. The addition of new glomeruli in the olfactory bulb during the postnatal period, although a somewhat controversial topic, has been reported in rats (LaMantia and Purves, 1989; Pomeroy et al., 1990). However, because we also found numerous microglomeruli in adult mice (P60), it seems unlikely that they represent newly forming glomeruli. Evidence is also lacking to suggest that microglomeruli are degenerating glomeruli. First, we are not aware of any data that suggest that glomeruli are lost over the course of development or in adults. Second, in light of the fact that the number of microglomeruli in perinatal mice (P6) appeared similar to the number seen in adults, the conclusion that microglomeruli represent degenerating glomeruli would be extraordinary, because it would suggest that $\sim 10 \%$ of $\mathrm{MOB}$ glomeruli are degenerating at any point in time. Thus, we believe that the most parsimonious conclusion is that the microglomeruli are a previously unrecognized organizational element of the olfactory bulb.

\section{Are UEA ${ }^{+}$processes mistargeted?}

One possible explanation for the presence of microglomeruli is that the $\mathrm{UEA}^{+}$axons, which innervate these structures, originate from a subset of OSNs that fail to properly express NCAM. Microglomeruli could represent a subset of OSN axons that are excluded from traditional MOB glomeruli because of a lack of NCAM expression, but nevertheless converge to form microglomeruli. Indeed, olfactory bulbs of homozygous NCAM-180 mutants exhibited irregularly shaped, small glomeruli (Treloar et al., 1997), although the severity of these defects decreased in mature mice. However, several factors argue against the hypothesis that $\mathrm{UEA}^{+}$axons are defective OSNs that fail to reach their proper targets. First, work in the NCAM-180 mutant suggests that the observed decrease in the size of glomeruli was attributable to a decrease in OSN axons leaving the ONL (Treloar et al., 1997). If $\mathrm{UEA}^{+}$processes are defective OSNs, we would expect them to have great difficulty leaving the ONL layer, especially in the presence of large numbers of competent OSNs. Second, the mature phenotype displayed by microglomeruli suggests that $\mathrm{UEA}^{+}$processes within microglomeruli are likely to be electrophysiologically active. Although activity appears to be unnecessary for the formation of neuropilar structures (Belluscio et al., 1998; Baker et al., 1999; Lin et al., 2000), the development of these structures is altered in the absence of electrophysiological activity. In particular, inactive glomeruli in the olfactory cyclic nucleotide gated channel 1 homozygous mutant fail to develop significant connections with interneurons (Baker et al., 1999). In contrast, dendritic innervation of microglomeruli by interneurons, as measured by GAD labeling, was equivalent to that seen in traditional MOB glomeruli (compare Fig. 6A,B). Finally, the convergence of fascicles and single axons observed in several microglomeruli (compare Figs. $1 B, 3 C$ ) suggests that $\mathrm{UEA}^{+}$ processes are being guided toward coalescence rather than randomly mistargeting. It is notable that the convergence of the $\mathrm{UEA}^{+}$and $\mathrm{OMP}^{+}$processes within the microglomeruli is quite dense, perhaps more so than occurs in the larger MOB glomeruli. The significance of this is unclear, but it suggests that the ratio of dendritic to axonal processes may be lower in microglomeruli than in MOB glomeruli.

It also seems unlikely that the microglomeruli are related to the small ectopic glomeruli-innervated axons from OSNs that express the P2 odor receptor (Royal and Key, 1999; Schaefer et al., 2001).
These differ in size from the larger P2-innervated glomeruli, but they do not show the consistent deep placement in the glomerular layer, proximal to the external plexiform layer, that is characteristic of microglomeruli.

The role of NCAM in axon guidance is unclear, but within the olfactory system NCAM mutants display unusual innervation of MOB glomeruli (Treloar et al., 1997) and a sharp decrease in the migration of interneurons and astrocytes from the rostral migratory stream (Tomasiewicz et al., 1993; Chazal et al., 2000). Throughout the nervous system, NCAM appears to play a role in promoting axon growth, axon targeting, and synapse formation (for review, see Walsh and Doherty, 1997; Doherty et al., 2000). The lack of NCAM labeling in microglomerular axons indicates that they are able to converge and target independently of NCAM expression. A developmental analysis of microglomeruli may provide some clues about the mechanisms that influence their formation relative to MOB glomeruli (Treloar et al., 1999)

\section{What are microglomeruli?}

Microglomeruli clearly represent a novel olfactory structure; however, their functional significance is unclear. One difficulty in understanding the function of microglomeruli is that the number and extent of microglomeruli in the MOB are still unclear. Although 100-150 $\mathrm{UEA}^{+}$microglomeruli were observed in a typical adult olfactory bulb, it remains a possibility that other microglomeruli are present that do not express a UEA binding epitope. For example, preliminary observations indicate that a subset of $\mathrm{UEA}^{+}$microglomeruli were also Dolichos biflorus agglutininpositive $\left(\mathrm{DBA}^{+}\right)$(data not shown). Although there are no data to indicate that these may constitute a second population of microglomeruli, it does raise the possibility that others may be present that were not detected by either UEA or DBA. The fact that microglomeruli have not been observed previously in mice is an indication of the difficulty of visualizing microglomeruli. On a standard or confocal microscope, in the absence of unique markers, these small neuropilar structures are nearly indistinguishable from adjacent glomeruli. A greater understanding of the molecular phenotype of microglomeruli will be necessary to make a final determination of the extent of microglomeruli in the MOB.

The role that microglomeruli play in the olfactory system of mice is primarily dependent on the odorant receptors expressed in $\mathrm{UEA}^{+}$microglomerular-innervating OSNs ( $\mu$ OSNs). One possibility is that $\mu$ OSNs express traditional G-protein-coupled odorant receptors (Buck and Axel, 1991; Levy et al., 1991; Ngai et al., 1993) and recognize a subset of volatile odorants. Indeed, $\mathrm{NCAM}^{-} \mu \mathrm{OSNs}$ in the MOE were morphologically similar to OSNs that innervate traditional glomeruli. However, given the relatively small sensory and GABAergic innervation of microglomeruli, it seems unlikely that $\mu$ OSNs could play a significant role in the first-order processing of odorant information as it is currently conceived (for review, see Laurent, 1999; Mori et al., 1999). Alternatively, $\mu$ OSNs could express receptors similar to those found in the sensory neurons of the vomeronasal organ (for review, see Keverne, 1999; Dulac, 2000). However, no vomeronasal receptors (VRs) have been found in the MOE (Herrada and Dulac, 1997; Matsunami and Buck, 1997; Ryba and Tirindelli, 1997; Pantages and Dulac, 2000), suggesting that if $\mu$ OSNs transduce pheromones, they do so with a previously unidentified family of VRs. The identification of the odorant receptor proteins expressed by $\mu$ OSNs will be critical in determining the mechanisms that underlie axon guidance. In the main olfactory system, odorant receptors are necessary for the guidance of axons to their correct glomerular target (Mombaerts et al., 1996; Wang et al., 1998). In the accessory olfactory system, deletion of an odorant 
receptor from a subset of OSNs leads to mistargeting in the AOB (Belluscio et al., 1999; Rodriguez et al., 1999). The odorants recognized by $\mu$ OSNs, the manner of signal transduction in these cells, the mechanisms by which their axons reach microglomeruli, and their physiological importance remain intriguing questions for additional investigation.

\section{REFERENCES}

Baker H, Grillo M, Margolis FL (1989) Biochemical and immunocytochemical characterization of olfactory marker protein in the rodent central nervous system. J Comp Neurol 285:246-261.

Baker H, Cummings DM, Munger SD, Margolis JW, Franzen L, Reed RR, Margolis FL (1999) Targeted deletion of a cyclic nucleotide-gated channel subunit (OCNC1): biochemical and morphological consequences in adult mice. J Neurosci 19:9313-9321.

Belluscio L, Gold GH, Nemes A, Axel R (1998) Mice deficient in G(olf) are anosmic. Neuron 20:69-81.

Belluscio L, Koentges G, Axel R, Dulac C (1999) A map of pheromone receptor activation in the mammalian brain. Cell 97:209-220.

Buck L, Axel R (1991) A novel multigene family may encode odorant receptors: a molecular basis for odor recognition. Cell 65:175-187.

Chazal G, Durbec P, Jankovski A, Rougon G, Cremer H (2000) Consequences of neural cell adhesion molecule deficiency on cell migration in the rostral migratory stream of the mouse. J Neurosci 20:1446-1457.

Doherty P, Williams G, Williams EJ (2000) CAMs and axonal growth: a critical evaluation of the role of calcium and the MAPK cascade. Mol Cell Neurosci 16:283-295.

Dulac C (2000) Sensory coding of pheromone signals in mammals. Curr Opin Neurobiol 10:511-518.

Gabellec MM, Recasens M, Benezra R, Mandel P (1980) Regional distributions of gamma-aminobutyric acid (GABA), glutamate decarboxylase (GAD), and gamma-aminobutyrate transaminase (GABA-T) in the central nervous brains of $\mathrm{C} 57 / \mathrm{BR}, \mathrm{C} 3 \mathrm{H} / \mathrm{He}$, and $\mathrm{F} 1$ hybrid mice. Neurochem Res 5:309-317.

Giannetti N, Saucier D, Astic L (1992) Organization of the septal organ projection to the main olfactory bulb in adult and newborn rats. J Comp Neurol 323:288-298.

Gong Q, Shipley MT (1996) Expression of extracellular matrix molecules and cell surface molecules in the olfactory nerve pathway during early development. J Comp Neurol 366:1-14.

Greer CA, Stewart WB, Teicher MH, Shepherd GM (1982) Functional development of the olfactory bulb and a unique glomerular complex in the neonatal rat. J Neurosci 2:1744-1759.

Herrada G, Dulac C (1997) A novel family of putative pheromone receptors in mammals with a topographically organized and sexually dimorphic distribution. Cell 90:763-773.

Imamura K, Mori K, Fujita SC, Obata K (1985) Immunochemical identification of subgroups of vomeronasal nerve fibers and their segregated terminations in the accessory olfactory bulb. Brain Res 328:362-366.

Juilfs DM, Fulle HJ, Zhao AZ, Houslay MD, Garbers DL, Beavo JA (1997) A subset of olfactory neurons that selectively express cGMPstimulated phosphodiesterase (PDE2) and guanylyl cyclase-D define a unique olfactory signal transduction pathway. Proc Natl Acad Sci USA 94:3388-3395

Kasowski HJ, Kim H, Greer CA (1999) Compartmental organization of the olfactory bulb glomerulus. J Comp Neurol 407:261-274.

Keverne EB (1999) The vomeronasal organ. Science 286:716-720.

Kim H, Greer CA (2000) The emergence of compartmental organization in olfactory bulb glomeruli during postnatal development. J Comp Neurol 422:297-311.

Kosaka K, Kosaka T (1999) Distinctive neuronal organization of the olfactory bulb of the laboratory shrew. NeuroReport 10:267-273.

Kosaka K, Kosaka T (2001) Nidus and tasseled cell: distinctive neuronal organization of the main olfactory bulb of the laboratory musk shrew (Suncus murinus). J Comp Neurol 430:542-561.

Kosaka K, Toida K, Aika Y, Kosaka T (1998) How simple is the organization of the olfactory glomerulus? The heterogeneity of so-called periglomerular cells. Neurosci Res 30:101-110.

LaMantia AS, Purves D (1989) Development of glomerular pattern visualized in the olfactory bulbs of living mice. Nature 341:646-649.

Laurent G (1999) A systems perspective on early olfactory coding. Science 286:723-728.

Lernmark A (1996) Glutamic acid decarboxylase: gene to antigen to disease. J Intern Med 240:259-277.

Levy NS, Bakalyar HA, Reed RR (1991) Signal transduction in olfactory neurons. J Steroid Biochem Mol Biol 39:633-637.

Lin DM, Wang F, Lowe G, Gold GH, Axel R, Ngai J, Brunet L (2000) Formation of precise connections in the olfactory bulb occurs in the absence of odorant-evoked neuronal activity. Neuron 26:69-80.

Margolis FL (1982) Olfactory marker protein (OMP). Scand J Immunol Suppl 9:181-199.
Matsunami H, Buck LB (1997) A multigene family encoding a diverse array of putative pheromone receptors in mammals. Cell 90:775-784.

Mombaerts P, Wang F, Dulac C, Chao SK, Nemes A, Mendelsohn M, Edmondson J, Axel R (1996) Visualizing an olfactory sensory map. Cell 87:675-686.

Mori K, Nagao H, Yoshihara Y (1999) The olfactory bulb: coding and processing of odor molecule information. Science 286:711-715.

Ngai J, Dowling MM, Buck L, Axel R, Chess A (1993) The family of genes encoding odorant receptors in the channel catfish. Cell 72:657-666.

Pantages E, Dulac C (2000) A novel family of candidate pheromone receptors in mammals. Neuron 28:835-845.

Pedersen PE, Benson TE (1986) Projection of septal organ receptor neurons to the main olfactory bulb in rats. J Comp Neurol 252:555-562.

Plendl J, Sinowatz F (1998) Glycobiology of the olfactory system. Acta Anat (Basel) 161:234-253.

Pomeroy SL, LaMantia AS, Purves D (1990) Postnatal construction of neural circuitry in the mouse olfactory bulb. J Neurosci 10:1952-1966.

Ressler KJ, Sullivan SL, Buck LB (1993) A zonal organization of odorant receptor gene expression in the olfactory epithelium. Cell 73:597-609.

Ressler KJ, Sullivan SL, Buck LB (1994) Information coding in the olfactory system: evidence for a stereotyped and highly organized epitope map in the olfactory bulb. Cell 79:1245-1255.

Ribak CE, Vaughn JE, Saito K, Barber R, Roberts E (1977) Glutamate decarboxylase localization in neurons of the olfactory bulb. Brain Res 126:1-18.

Ring G, Mezza RC, Schwob JE (1997) Immunohistochemical identification of discrete subsets of rat olfactory neurons and the glomeruli that they innervate. J Comp Neurol 388:415-434.

Risser JM, Slotnick BM (1987) Suckling behavior in rat pups with lesions which destroy the modified glomerular complex. Brain Res Bull 19:275-281.

Rodriguez I, Feinstein P, Mombaerts P (1999) Variable patterns of axonal projections of sensory neurons in the mouse vomeronasal system. Cell 97:199-208.

Royal SJ, Key B (1999) Development of P2 olfactory glomeruli in P2internal ribosome entry site-tau-LacZ transgenic mice. J Neurosci 19:9856-9864.

Royet JP, Souchier C, Jourdan F, Ploye H (1998) Morphometric study of the glomerular population in the mouse olfactory bulb: numerical density and size distribution along the rostrocaudal axis. J Comp Neurol 270:559-568.

Ryba NJ, Tirindelli R (1997) A new multigene family of putative pheromone receptors. Neuron 19:371-379.

Schaefer ML, Finger TE, Restrepo D (2001) Variability of position of the P2 glomerulus within a map of the mouse olfactory bulb. J Comp Neurol 436:351-362.

Shinoda K, Shiotani Y, Osawa Y (1989) Necklace olfactory glomeruli form unique components of the rat primary olfactory system. J Comp Neurol 284:362-373.

Shinoda K, Ohtsuki T, Nagano M, Okumura T (1993) A possible functional necklace formed by placental antigen X-P2-immunoreactive and intensely acetylcholinesterase-reactive (PAX/IAE) glomerular complexes in the rat olfactory bulb. Brain Res 618:160-166.

Terkelsen OB, Bock E, Mollgard K (1989) NCAM and Thy-1 in special sense organs of the developing mouse. Anat Embryol (Berl) 179:311-318.

Tomasiewicz H, Ono K, Yee D, Thompson C, Goridis C, Rutishauser U, Magnuson T (1993) Genetic deletion of a neural cell adhesion molecule variant (N-CAM-180) produces distinct defects in the central nervous system. Neuron 11:1163-1174.

Treloar H, Tomasiewicz H, Magnuson T, Key B (1997) The central pathway of primary olfactory axons is abnormal in mice lacking the N-CAM-180 isoform. J Neurobiol 32:643-658.

Treloar HB, Purcell AL, Greer CA (1999) Glomerular formation in the developing rat olfactory bulb. J Comp Neurol 413:289-304.

Valverde F, Santacana M, Heredia M (1992) Formation of an olfactory glomerulus: morphological aspects of development and organization. Neuroscience 49:255-275

Vassar R, Ngai J, Axel R (1993) Spatial segregation of odorant receptor expression in the mammalian olfactory epithelium. Cell 74:309-318.

Vassar R, Chao SK, Sitcheran R, Nunez JM, Vosshall LB, Axel R (1994) Topographic organization of sensory projections to the olfactory bulb. Cell 79:981-991.

Walsh FS, Doherty P (1997) Neural cell adhesion molecules of the immunoglobulin superfamily: role in axon growth and guidance. Annu Rev Cell Dev Biol 13:425-456.

Wang F, Nemes A, Mendelsohn M, Axel R (1998) Odorant receptors govern the formation of a precise topographic map. Cell 93:47-60.

Weruaga E, Brinon JG, Porteros A, Arevalo R, Aijon J, Alonso JR (2001) A sexually dimorphic group of atypical glomeruli in the mouse olfactory bulb. Chem Senses 26:7-15.

Yamashita H, Kawata K, Takahashi M (1998) Upregulation of neural growth-associated protein and neural cell adhesion molecule in mouse olfactory epithelium and axons after unilateral removal of the olfactory bulb. Eur Arch Otorhinolaryngol 255:441-445. 